\title{
EFEITOS DE OFICINA EDUCATIVA SOBRE PREVENÇÃO E CUIDADOS À CRIANÇA COM ENGASGO: ESTUDO DE INTERVENÇÃO
}

\section{EFFECTS OF AN EDUCATIONAL WORKSHOP ABOUT PREVENTION AND CARE OF CHOKING IN CHILDREN: AN INTERVENTION STUDY}

\section{EFECTOS DEL TALLER EDUCATIVO SOBRE PREVENCIÓN Y CUIDADO DEL ATRAGANTAMIENTO EN NIÑOS: ESTUDIO DE INTERVENCIÓN}

\author{
Priscila Costa ${ }^{1}$, Leticia Soares Silva ${ }^{2}$, Mariah Tomé Silva ${ }^{3}$, Claudia Maria de Freitas Floriano ${ }^{4}$, Kelly Christina Sbampato Calado Orsi ${ }^{5}$
}

\section{RESUMO}

Objetivo: Avaliar o efeito de uma oficina educativa sobre prevenção e cuidado à criança com engasgo no conhecimento de profissionais da saúde e da educação infantil. Métodos: Estudo quase-experimental com pré e pós-teste, e abordagem quantitativa conduzido em um centro assistencial filantrópico e uma escola de educação infantil. A intervenção foi composta, por oficina educativa teórico-prática, sobre prevenção e atendimento à criança com engasgo. O conhecimento dos participantes foi avaliado, por meio de questionário com nove itens antes e depois da intervenção. Resultados: Participaram do estudo 36 indivíduos, sendo 22 profissionais da educação infantil e 14 profissionais da saúde. Verificou-se aumento de $29,9 \%$ no conhecimento dos participantes após a oficina. A média de acertos no questionário passou de 5,7 para $8,3 \mathrm{com}$ diferença significativa $(p<0.01)$. Conclusão: As oficinas educativas ampliaram o conhecimento dos profissionais da saúde e educação infantil na prevenção e atendimento à criança com engasgo, destacando o cuidado à saúde da criança, por meio de ações intersetoriais.

Descritores: Primeiros Socorros; Saúde da Criança; Educação em Saúde; Enfermagem Pediátrica.

\begin{abstract}
Objective: to evaluate the effect of an educational workshop on prevention and care for choking children on the knowledge of health professionals and early childhood educators. Methods: a quasi-experimental study with pre and post-test, and a quantitative approach conducted in a philanthropic assistance center and a school for early childhood education. The intervention consisted of a theoretical-practical educational workshop on prevention and care for choking children . The participants' knowledge was assessed using a questionnaire with nine items before and after the intervention. Results: 36 individuals participated in the study: 22 childhood educators and 14 health professionals. There was a $29.9 \%$ increase in participants' knowledge after the workshop. The average number of correct answers in the questionnaire went from 5.7 to 8.3, with a significant difference $(p<0.01)$. Conclusion: the educational workshops expanded the knowledge of health professionals and early childhood educators in the prevention and care of choking children, highlighting the role in child health care through intersectoral actions.
\end{abstract}

Descriptors: First Aid; Child Health; Health Education; Pediatric Nursing.

\section{RESUMEN}

Objetivo: evaluar el efecto de un taller educativo sobre prevención y cuidado de niños con atragantamiento en el conocimiento de los profesionales de la salud y la educación de la primera infancia. Métodos: estudio casi experimental con pre y posprueba, y enfoque cuantitativo realizado en un centro de asistencia filantrópica y una escuela de educación infantil. La intervención consistió en un taller educativo teórico-práctico sobre prevención y cuidado de niños con atragantamiento. El conocimiento de los participantes se evaluó mediante un cuestionario con nueve ítems antes y después de la intervención. Resultados: 36 personas participaron en el estudio, 22 de ellas de educación infantil y 14 de profesionales de la salud. Hubo un aumento del $29,9 \%$ en el conocimiento de los participantes después del taller. El número promedio de respuestas correctas en el cuestionario pasó de 5.7 a 8.3, con una diferencia significativa ( $p<0.01)$. Conclusión: los talleres educativos ampliaron el conocimiento de los profesionales de la salud y la educación de la primera infancia para prevenir y ayudar a los niños con atragantamiento, destacando el papel de las enfermeras en la atención de la salud infantil a través de acciones intersectoriales.

Descriptores: Primeros Auxilios; Salud del Niño; Educación en Salud; Enfermería Pediátrica.

${ }^{1}$ Doutorado em Ciências da Saúde pela Universidade de São Paulo, Professora do Departamento de Enfermagem Pediátrica da Escola Paulista de Enfermagem da Universidade Federal de São Paulo. ${ }^{2}$ Enfermeira do Hospital São Paulo da Universidade Federal de São Paulo. ${ }^{3}$ Graduação em Enfermagem pela Universidade Federal de São Paulo. ${ }^{4}$ Doutoranda e Técnica Administrativa em Educação do Departamento de Enfermagem Pediátrica, Escola Paulista de Enfermagem, da Universidade Federal de São Paulo. ${ }^{5}$ Doutorado pela Universidade Federal de São Paulo, Técnica Administrativa em Educação do Departamento de Enfermagem Pediátrica, Escola Paulista de Enfermagem, Universidade Federal de São Paulo.

Como citar este artigo:

Costa $\mathrm{P}$, Silva LS, Silva MT, et al. Efeitos de oficina educativa sobre prevenção e cuidados à criança com engasgo: estudo de intervenção. Revista de Enfermagem do Centro-Oeste Mineiro. 2020;10:e3911. [Access http://doi.org/10.19175/recom.v10i0.3911 ]; Available in: DOI: 


\section{INTRODUÇÃO}

A obstrução de vias aéreas por corpo estranho (Ovace) ou engasgo representa uma das principais causas de mortalidade infantil, afetando, principalmente, crianças de um a três anos $^{(1)}$. Trata-se de um evento causado pela oclusão parcial ou completa da passagem de ar entre as vias aéreas superiores e a traqueia por um corpo estranho como alimento, moeda, bexiga ou brinquedo ${ }^{(1)}$.

No Brasil, a obstrução das vias aéreas por um corpo estranho representa grave problema de saúde pública na população pediátrica ${ }^{(2)}$. Em 2016, a sufocação foi a terceira causa de morte por acidentes entre crianças e adolescentes no país ${ }^{(3)}$. As características próprias do desenvolvimento da criança, nos primeiros anos de vida, tais como a exploração do mundo, através da via oral, a pequena distância entre os dentes incisivos e a base da língua, a maior frequência respiratória e o pequeno calibre das vias aéreas aumentam o risco desse agravo, nesta população(2).

Para a promoção e proteção da saúde da criança, o suporte básico de vida pediátrico deve ser parte de um esforço tanto da sociedade quanto de programas e políticas públicas que invistam no aprimoramento do conhecimento de profissionais da saúde e de pessoas leigas a exemplo dos profissionais da educação infantil quanto à prevenção e atendimento à criança vítima de Ovace ou parada cardiopulmonar. Assim, é essencial capacitar a população quanto à prevenção, manobras de desobstrução de vias aéreas, reanimação cardiopulmonar imediata se necessária, e acesso aos serviços de emergência ${ }^{(4)}$.

Estudo realizado para avaliar 0 conhecimento e habilidade sobre reanimação cardiopulmonar antes e após uma capacitação em suporte básico de vida com médicos e enfermeiros que atuam na atenção primária, revelou que somente $37,5 \%$ dos 32 profissionais enfermeiros e médicos já haviam participado de alguma capacitação de urgência/emergência e apenas 21,9\% tinham experiências satisfatórias no atendimento a vítimas de parada cardiorrespiratória ${ }^{(5)}$. O conhecimento sobre suporte básico de vida para lactentes e crianças é essencial para os profissionais de saúde e educação, destacando habilidades específicas para o atendimento da população pediátrica garantindo a abertura de vias aéreas, ventilação e circulação efetivas ${ }^{(6)}$. Logo, é necessário investir na educação permanente de profissionais da saúde e da educação visando o aprimoramento de seus conhecimentos e habilidades em suporte básico de vida, em especial na prevenção e atendimento à criança vítima de Ovace e consequente parada cardiopulmonar.

Nesse sentido, o consenso internacional ${ }^{(7)}$ de especialistas revelou que os itens essenciais a serem avaliados no conhecimento de pessoas leigas sobre o atendimento à vítima de Ovace incluem a identificação dos diferentes estágios da obstrução, avaliação do nível de consciência, como chamar por ajuda, como realizar a técnica de golpes nas costas e compressões torácicas ou abdominais, de acordo com a idade da criança, identificar a perda de consciência, iniciar a reanimação cardiopulmonar, avaliar a respiração e ventilar a vítima ${ }^{(7)}$. As práticas de simulação são válidas e confiáveis para o ensino e avaliação destas habilidades em pessoas leigas ${ }^{(8)}$ e profissionais da saúde ${ }^{(9)}$. A simulação realística representa um método de ensino eficaz para a aquisição de conhecimentos, além de promover a autoconfiança e satisfação como método de ensino dentre profissionais e estudantes da área da saúde ${ }^{(9)}$.

Corroborando as evidências quanto aos itens essenciais a serem avaliados no conhecimento relacionado à desobstrução de vias aéreas por corpo estranho e considerando a simulação de atendimento uma forma válida e confiável para o ensino no tema, no presente estudo, objetivou-se avaliar o efeito de uma oficina educativa, no conhecimento de profissionais da saúde e da educação infantil sobre prevenção e cuidado à criança vítima de engasgo ou obstrução de vias aéreas por corpo estranho.

\section{MÉTODOS}

Trata-se de um estudo quase experimental com pré e pós-teste e abordagem quantitativa. $\mathrm{O}$ estudo foi conduzido de agosto a outubro de 2017, em uma escola pública de educação infantil e em um ambulatório de atenção primária em saúde de um centro assistencial de caráter filantrópico, que atende, prioritariamente, famílias com crianças na primeira infância e gestantes, ambos localizados no município de São Paulo. A demanda pelas oficinas educativas partiu dos profissionais da educação infantil e da direção do ambulatório de saúde.

As oficinas educativas foram realizadas nos locais de trabalho dos profissionais, tendo a participação de um a dez profissionais por oficina. Cada sujeito participou de uma oficina, que foi realizada em seu local de trabalho em diferentes 
dias e horários, com a mesma estrutura e conteúdo, para que, a maioria dos profissionais pudesse participar.

A amostra, de conveniência, foi composta por profissionais da saúde e da educação. Os critérios de inclusão foram ser profissional da saúde ou da educação atuante no centro assistencial ou na escola de educação infantil. 0 critério de exclusão adotado foi não participar da oficina educativa, após o preenchimento do questionário de avaliação de conhecimentos relacionado ao tema.

A oficina teve duração de 60 a 90 minutos e foi dividida em duas etapas. Na primeira etapa, de aproximadamente 30 minutos, foi realizada uma roda de conversa e exposição dialogada sobre o tema, buscando refletir junto aos participantes sobre aspectos que eles conheciam acerca do tema e compartilhar conhecimento. O recurso midiático utilizando para esta etapa foi desenvolvido pelos autores de acordo com a recomendação da Sociedade Brasileira de Cardiologia $^{(10)}$ e da American Heart Association ${ }^{(4)}$.

O conteúdo apresentado, nesta etapa, incluiu a epidemiologia das mortes por sufocação no Brasil, suas principais causas e estratégias de prevenção no domicílio e na escola, a identificação dos sinais de obstrução parcial e total em crianças, a sequência de atendimento incluindo as manobras de desobstrução de vias aéreas em lactentes e em crianças maiores de um ano até a puberdade, telefones e serviços para chamar por ajuda, a avaliação do nível de consciência e as manobras de reanimação cardiopulmonar para os casos em que a criança perde a consciência, assim como o que não fazer com a criança que teve engasgo. Enquanto os conteúdos eram discutidos com os participantes, uma demonstração das manobras, utilizando manequins infantis, era realizada.

A segunda etapa da oficina envolveu a simulação do atendimento, utilizando manequins infantis que estavam dispostos em colchonetes no chão. Cada pesquisador demonstrou o atendimento e, em seguida, supervisionou uma dupla de participantes. Essa etapa teve duração aproximada de 30 à 60 minutos, permitindo o compartilhamento de saberes e a simulação do atendimento mais de uma vez por participante.

Ao final da oficina educativa, foram distribuídos aos participantes folhetos educativos e folders, elaborados pela equipe de pesquisa, que traziam o conteúdo teórico-prático abordado na oficina educativa.
As variáveis de caracterização dos participantes incluíram idade, sexo, ocupação, tempo de experiência profissional e participação prévia em capacitação sobre o tema. A intervenção foi representada pela oficina educativa, e como se trata se estudo quaseexperimental, todos os sujeitos do estudo foram submetidos à intervenção.

A variável dependente foi o conhecimento dos participantes sobre prevenção e atendimento à criança vítima de Ovace. Tal conhecimento foi avaliado uma semana antes e uma semana depois da oficina educativa, por meio de questionário composto por nove itens. Os nove itens do questionário que avaliou o conhecimento dos participantes versaram sobre a definição, estratégias de prevenção e identificação dos sinais de Ovace, manobras de desobstrução de vias aéreas em lactentes e em crianças maiores de um ano até a puberdade, a avaliação do nível de consciência para reconhecimento de uma parada cardiopulmonar, e as manobras de reanimação cardiopulmonar para lactentes e para crianças maiores de um ano até a puberdade.

O questionário sobre os conhecimentos dos participantes a respeito do tema foi elaborado pelos pesquisadores e validado por três juízes leigos e três especialistas. O questionário obteve grau de concordância de 91,2\%, calculado utilizando-se o percentual de concordância absoluta entre os juízes (número de concordâncias dividido pelo número total de avaliações). Valores superiores a $70 \%$ indicam bom grau de consenso entre os juízes ${ }^{(11)}$. Os juízes leigos avaliaram os enunciados e as assertivas de múltipla escolha quanto à clareza na linguagem. Os juízes foram escolhidos de forma que representassem ocupações frequentes em escolas de educação infantil e serviços de saúde e que lidassem diretamente com o público, tais como secretária executiva, professor e segurança.

A validação do questionário pelos juízes especialistas foi realizada quanto à clareza na linguagem e à descrição correta do item avaliado. Em relação à profissão dos juízes especialistas, todos eram enfermeiros, sendo que um deles era enfermeiro assistencial de um pronto-socorro adulto e infantil de um hospital público, na cidade de São Paulo e instrutor do suporte básico de vida. O segundo era enfermeiro, especialista em centro cirúrgico e professor de uma escola técnica de Enfermagem de um hospital privado de grande porte em São Paulo. O terceiro juiz era enfermeiro, mestre e doutor em Ciências, docente 
do Departamento de Enfermagem Pediátrica de uma universidade pública federal em São Paulo.

Os dados foram armazenados em planilha do programa Excel da Microsoft e analisados no software R. As variáveis categóricas foram apresentadas segundo frequências absoluta e relativa, e as variáveis numéricas, segundo estatística descritiva com média, desvio-padrão, mínimo e máximo. A normalidade da distribuição dos dados foi analisada pela distribuição da assimetria e curtose da curva, considerando os valores normais entre -2 e 2 . Para análises de associação, foi considerado nível de significância de $5 \%$, e realizados os testes de Wilcoxon ${ }^{(\mathrm{TW})}$, Exato de Fisher e Qui-quadrado.

O projeto foi aprovado pelo Comitê de Ética e Pesquisa da Universidade Federal de São Paulo (CAAE: 71271417.0.0000.5505) e atende às recomendações da Resolução CNS 466/2012.
Todos os participantes foram esclarecidos e assinaram o Termo de Consentimento Livre e Esclarecido.

\section{RESULTADOS}

Participaram do estudo 36 profissionais, destes 14 (38,9\%) eram profissionais da saúde atuantes em um centro assistencial filantrópico e 22 (61,1\%) eram profissionais da educação atuantes em uma escola pública de educação infantil.

Quanto às características dos participantes, a média de idade foi de 42,4 anos, com mínimo de 20 anos e máximo de 67 anos. Em relação ao tempo de experiência profissional, a média foi de 15,6 anos, variando de um a mais de 40 anos. Os demais dados de caracterização dos participantes são apresentados na Tabela 1.

Tabela 1 - Caracterização dos participantes do estudo quanto ao sexo, ocupação, capacitação e vivências prévias. São Paulo (SP) - Brasil, 2017.

\begin{tabular}{|c|c|c|}
\hline Características dos participantes & $\mathbf{N}$ & $\%$ \\
\hline \multicolumn{3}{|l|}{$\operatorname{Sexo}(n=36)$} \\
\hline Feminino & 24 & 66 \\
\hline Masculino & 12 & 34 \\
\hline \multicolumn{3}{|l|}{ Ocupação dos profissionais da saúde ( $n=14)$} \\
\hline Enfermeiro & 4 & 28,6 \\
\hline Dentista & 3 & 21,4 \\
\hline Nutricionista & 2 & 14,3 \\
\hline Técnico em enfermagem & 2 & 14,3 \\
\hline Assistente social & 1 & 7,1 \\
\hline Auxiliar de nutrição & 1 & 7,1 \\
\hline Farmacêutico & 1 & 7,1 \\
\hline \multicolumn{3}{|l|}{ Ocupação dos profissionais da educação (n=22) } \\
\hline Professor & 5 & 22,7 \\
\hline Serviço de apoio & 8 & 36.4 \\
\hline Auxiliar de pedagogia & 3 & 13,6 \\
\hline Gerente ou auxiliar administrativo, secretário executivo & 5 & 22,7 \\
\hline Não especificado & 1 & 4,5 \\
\hline \multicolumn{3}{|l|}{ Capacitação prévia sobre Ovace $(n=36)$} \\
\hline Sim & 2 & 5,6 \\
\hline Não & 34 & 94,4 \\
\hline \multicolumn{3}{|l|}{ Capacitação prévia sobre reanimação cardiopulmonar ( $n=36$ ) } \\
\hline Sim & 7 & 19,4 \\
\hline Não & 29 & 80,6 \\
\hline \multicolumn{3}{|l|}{ Vivência prévia em socorrer uma vítima de Ovace $(n=36)$} \\
\hline $\operatorname{Sim}$ & 4 & 11,1 \\
\hline Não & 32 & 88,8 \\
\hline \multicolumn{3}{|l|}{ Vivência prévia em socorrer uma vítima em PCR ( $n=36$ ) } \\
\hline Sim & 1 & 2,7 \\
\hline Não & 35 & 97,3 \\
\hline
\end{tabular}

Legenda: PCR - parada cardiorespiratória. Fonte: Dados da pesquisa.

A minoria dos participantes possuía capacitação prévia sobre o tema ou vivência no atendimento a uma vítima de engasgo ou parada cardiopulmonar. Verificou-se, ainda, que os dois profissionais que tinham essa capacitação, um era enfermeiro e o outro dentista, e dentre os sete que já haviam realizado a capacitação, cinco eram profissionais da saúde, sendo dois dentistas, um enfermeiro, um técnico em enfermagem e um nutricionista, e dois eram profissionais da 
educação, sendo um professor e um recepcionista da escola. Dentre os participantes que relataram ter socorrido vítimas de engasgo, dois eram enfermeiros, um era professor e um era administrador da escola. $O$ único profissional que afirmou ter socorrido uma vítima de parada cardiopulmonar foi um enfermeiro.

Em relação à avaliação dos participantes sobre a oficina educativa, $33(91,6 \%)$ avaliaram como excelente e $3(8,3 \%)$ como boa. Todos ( $n=36 / 100,0 \%)$ consideraram a temática importante. Em relação ao efeito da oficina educativa no conhecimento dos participantes sobre a prevenção e atendimento à criança vítima de engasgo, verificou-se que a média de acertos no questionário antes da oficina foi de 5,7 e após foi de 8,3 com diferença estatisticamente significante $(p=0,000003)$.

Tabela 2 - Distribuição de acertos dos profissionais antes e após as oficinas educativas sobre atendimento a crianças vítimas de obstrução de vias aéreas por corpo estranho. São Paulo (SP) - Brasil, 2017.

\begin{tabular}{|c|c|c|c|c|}
\hline Questões & $\begin{array}{l}\text { Acerto antes da } \\
\text { intervenção } \\
\mathbf{n}(\%) \\
\end{array}$ & $\begin{array}{l}\text { Acerto após a } \\
\text { intervenção } \\
\mathrm{n}(\%)\end{array}$ & $\begin{array}{l}\text { Porcentagem } \\
\text { de incremento } \\
\%\end{array}$ & Valor de $p$ \\
\hline \multicolumn{5}{|l|}{ Questão 1. O que é engasgo? } \\
\hline $\begin{array}{l}\text { Resposta: Engasgo é quando um alimento ou outro objeto } \\
\text { fica preso na garganta bloqueando a passagem do ar para } \\
\text { os pulmões } \\
\text { Questão 2. Como evitar o engasgo em crianças? }\end{array}$ & $30(83,3)$ & $32(88,9)$ & 5,6 & $0,74^{\mathrm{F}}$ \\
\hline $\begin{array}{l}\text { Resposta: Oferecer os alimentos cortados ou amassados } \\
\text { em pedaços pequenos }\end{array}$ & $33(91,7)$ & $36(100,0)$ & 8,3 & $0,24^{F}$ \\
\hline $\begin{array}{l}\text { Questão } 3 \text {. São sinais de que a criança está } \\
\text { completamente engasgada: } \\
\text { Resposta: A criança não consegue tossir ou chorar e os } \\
\text { lábios estão roxos. }\end{array}$ & $33(91,7)$ & $36(100,0)$ & 8,3 & $0,24^{F}$ \\
\hline \multicolumn{5}{|l|}{$\begin{array}{l}\text { Questão 4. As manobras para desengasgar um bebê } \\
\text { menor de um ano são: }\end{array}$} \\
\hline $\begin{array}{l}\text { Resposta: Dar } 5 \text { golpes no meio das costas do bebê e fazer } \\
5 \text { compressões torácicas com os dois dedos no meio do } \\
\text { peito }\end{array}$ & $17(47,2)$ & $32(88,9)$ & 41,7 & $0,0003^{F}$ \\
\hline \multicolumn{5}{|l|}{$\begin{array}{l}\text { Questão 5. As manobras para desengasgar crianças com } \\
\text { idade superior a um ano até a puberdade são: }\end{array}$} \\
\hline $\begin{array}{l}\text { Resposta: Abraçar a criança por trás, fechar uma das mãos } \\
\text { e agarrar a mão fechada com a outra mão, depois fazer } \\
\text { compressões rápidas acima do umbigo da criança. }\end{array}$ & $21(58,3)$ & $31(86,1)$ & 27,8 & $0,017^{F}$ \\
\hline $\begin{array}{l}\text { Questão 6. São sinais de que uma criança está em parada } \\
\text { cardiorrespiratória: } \\
\text { Resposta: A criança não responde (não chora, não se } \\
\text { mexe) e não respira. }\end{array}$ & $32(88,9)$ & $36(100,0)$ & 11,1 & $0,115^{\mathrm{F}}$ \\
\hline $\begin{array}{l}\text { Questão 7. Para socorrer uma criança em parada } \\
\text { cardiorrespiratória, recomenda-se: } \\
\text { Resposta: Realizar } 30 \text { compressões torácicas e } 02 \text { ventilações }\end{array}$ & $9(25,0)$ & $33(86,4)$ & 61,4 & $0,0001^{F}$ \\
\hline $\begin{array}{l}\text { Questão 8. Para realizar as compressões torácicas em um } \\
\text { bebê menor de um ano em parada cardiorrespiratória } \\
\text { deve-se: } \\
\text { Resposta: Colocar dois dedos no meio do peito, logo abaixo } \\
\text { da linha dos mamilos }\end{array}$ & $19(36,4)$ & $32(88,9)$ & 52,5 & $0,0007^{F}$ \\
\hline $\begin{array}{l}\text { Questão 9. Para realizar as compressões torácicas em } \\
\text { crianças com idade superior a um ano até a puberdade } \\
\text { deve-se: } \\
\text { Resposta: Colocar a palma de uma mão, logo abaixo da } \\
\text { linha dos mamilos. }\end{array}$ & $10(27,7)$ & $29(80,6)$ & 52,9 & $<0,001^{Q}$ \\
\hline
\end{tabular}

Legenda: F- Exato de Fisher; ${ }^{Q}$ - Qui-quadrado. Fonte: Dados da pesquisa.

Nos dados da Tabela 2, percebe-se que o conhecimento dos participantes apresentou incremento de $29,9 \%$, em média, considerando as nove questões avaliadas. Os conhecimentos com o maior número de acertos dos participantes, após a oficina educativa, foram aqueles relativos às manobras para socorrer a criança com obstrução das vias aéreas e a vítima de parada cardiopulmonar. Verificou-se maior incremento do conhecimento quanto à relação entre o número de compressões torácicas e o número de ventilações de resgate para um socorrista, 
atendendo a uma vítima de parada cardiopulmonar, bem como quanto às manobras de compressão cardíaca nas crianças menores de um ano e nas maiores de um ano até a puberdade.

Em relação ao conhecimento dos profissionais de saúde e da educação sobre a prevenção e atendimento da criança com OVACE, antes da intervenção, a média de acertos entre os profissionais da saúde foi de 6,6 e dos profissionais da educação foi de 5,0 , sendo que esse resultado foi, estatisticamente, significante $(p=0,001)$. Após a intervenção, a média de acertos entre os profissionais da saúde foi de 8,6 , e educadores, 8,0 , sendo que esse resultado não foi, estatisticamente, significante $(p=0,32)$

\section{DISCUSSÃO}

Os achados do presente estudo revelaram que as oficinas educativas contribuíram para a ampliação de conhecimento dos profissionais de saúde e da educação sobre a prevenção e atendimento da criança vítima de obstrução de vias aéreas por corpo estranho. Após as oficinas, 0 conhecimento dos participantes sobre o tema aumentou, aproximadamente, 30\%, especialmente relacionado ao atendimento da criança que perde a consciência, em decorrência da OVACE e necessita, então, de reanimação cardiopulmonar. Esses achados destacam o papel do enfermeiro como educador em saúde e articulador de ações intersetoriais, para a proteção e promoção da saúde da criança.

Resultados semelhantes foram reportados em estudo realizado com profissionais da área de enfermagem que avaliou um treinamento teóricoprático sobre suporte básico de vida, revelando que o conhecimento dos profissionais passou de 4,1 pontos para 7,3 pontos após o treinamento, aumentando $33 \%{ }^{(12)}$. Outro estudo que objetivou comparar o conhecimento antes e após um minicurso de 20 horas sobre manobras de atendimento pré-hospitalar em suporte básico de vida para recém-ingressantes de uma universidade pública de medicina mostrou que a média de acertos passou de 20,1 para 24,3 com diferença, estatisticamente, significante $(p<0,01)$. Quanto às questões relacionadas ao atendimento inicial de vítimas de acidentes e urgências domiciliares, houve um incremento de $42,2 \%$ após a formação(13).

Quanto à importância do investimento em ações intersetoriais, estudo desenvolvido por graduandos de Enfermagem que realizaram oficinas educativas com 10 professores da educação primária de uma escola municipal, no Pará, identificou que os educadores não estavam preparados para agir em situações de risco à saúde da criança, incluindo o engasgo. Após rodas de conversa e práticas baseadas no protocolo de Suporte Básico de Vida, os educadores sentiramse acolhidos e capazes para o enfrentamento de tais situações. Assim, destacamos que parcerias entre os profissionais da saúde e da educação são promissoras no âmbito da prevenção de acidentes no ambiente escolar ${ }^{(14)}$.

A literatura aponta que o conhecimento teórico em primeiros socorros é importante para que os profissionais reconheçam os sinais e sintomas apresentados por uma criança com engasgo ou parada cardiopulmonar e realizem as manobras de maneira efetiva. Além disso, sabe-se que o conhecimento e a experiência estão diretamente relacionados com a sobrevida dos pacientes vítimas de engasgo e/ou parada cardiopulmonar. No entanto, o investimento na capacitação dos profissionais para esses atendimentos ainda não é prioritário e satisfatório ${ }^{(5,12,15)}$.

Nesse sentido, em 2004 a American Heart Association recomendou a inclusão do suporte básico de vida no currículo escolar de alunos do ensino médio e que fossem estabelecidas metas, para que todos os professores e estudantes recebessem treinamento em reanimação cardiopulmonar em consonância com as orientações da International Liaison committee on Resuscitation $^{(16)}$. No entanto, no Brasil, ainda não há uma legislação que assegure a incorporação desse conteúdo como parte obrigatória do currículo escolar ${ }^{(17)}$.

Há evidências de que profissionais médicos, enfermeiros e fisioterapeutas possuam conhecimento insuficiente em relação ao suporte básico de vida, particularmente relacionado ao reconhecimento de uma parada cardiopulmonar, retardando o tempo de resposta e o início das manobras de reanimação cardiopulmonar. Além disso, a falta de atualização para o atendimento da parada cardiopulmonar é comum entre os profissionais que não lidam, frequentemente, com essa situação, o que influencia na agilidade de identificar a situação e qualidade do atendimento $^{(18-19)}$. Porém, nossos resultados mostraram que o conhecimento dos profissionais da saúde antes da intervenção era maior que a dos profissionais da educação, demonstrando que há perpetuação do conhecimento, apesar de a média de acerto ser de 6,6, e que após a intervenção, 
tanto profissionais da saúde como da educação obtiveram, médias de acerto próximas, igualando o conhecimento dos profissionais, independentemente de serem da saúde e já terem passado por treinamentos prévios.

Um exemplo de iniciativa para disseminação do conhecimento a respeito do tema inclui um programa de educação em reanimação cardiorrespiratória de um projeto de extensão da Universidade Federal do Ceará, fundado em 1996, com o objetivo de ampliar o conhecimento acerca da parada cardiopulmonar a todos os profissionais, acadêmicos e leigos, por meio de aulas teórico-práticas ${ }^{(20)}$.

Nossos resultados apontam para a necessidade de fortalecer os profissionais atuantes nos serviços de saúde e no ambiente escolar quanto aos seus conhecimentos e habilidades na prevenção e atendimento à criança vítima de OVACE. Nesse sentido, a educação permanente em saúde é construída a partir do conhecimento do ambiente de trabalho e problemas diários encontrados na realidade, superando lacunas e melhorando o processo de trabalho em saúde. A educação permanente em saúde tem como objetivo a transformação das práticas profissionais e da própria organização do trabalho. Além disso, suas bases são as necessidades de saúde das pessoas e das populações, controle social em saúde e reorganização da gestão setorial(20).

Estudos conduzidos em outros países demonstraram a importância da educação permanente relacionada ao suporte básico de vida $^{(18-19)}$. Estudo retrospectivo realizado em Tuzla, Bósnia e Herzegovina, avaliou 772 prontuários e identificou que as principais complicações decorrentes dos engasgos estão relacionadas ao diagnóstico tardio e errôneo. Os autores recomendam a realização de medidas educativas, como campanhas de educação pública, para a prevenção desse agravo e diminuição da mortalidade relacionada ao engasgo ${ }^{(21)}$.

Algumas limitações do presente estudo envolvem a pequena amostra e não realização de outros testes, após a intervenção para avaliação da retenção do conteúdo. Contudo, o estudo inova, ao propor uma intervenção que promove a educação permanente de profissionais da saúde e da educação quanto ao atendimento à criança vítima de OVACE.

\section{CONCLUSÃO}

Este trabalho gerou evidências sobre a efetividade de uma intervenção educativa intersetorial voltada à promoção da saúde da criança. O conhecimento de profissionais da saúde e da educação infantil sobre prevenção e atendimento à criança vítima de obstrução de vias aéreas, por corpo estranho, aumentou, após oficina educativa, sendo a média de acertos das perguntas do questionário, significativamente, maior após a intervenção.

A realização de oficinas teórico-práticas sobre a prevenção e atendimento à criança com obstrução de vias aéreas, por corpo estranho, representa uma estratégia eficaz na educação permanente de profissionais da saúde e da educação, para que possam contribuir na promoção e proteção da saúde da criança. Portanto, políticas e programas públicos de caráter intersetorial são necessários para a disseminação de conhecimento relacionado ao tema para a sociedade.

\section{REFERÊNCIAS}

1- Duckett SA, Roten RA. Choking. Treasure Island: StatPearls; 2019.

2- Gikas RMC. Aspiração/ingestão de corpos estranhos. São Paulo: Sociedade de Pediatria de São Paulo; 2016.

3- Ministério da Saúde (BR). Estatísticas vitais: Óbitos por causas externas. Portal da Saúde 2016 [citado em 22 mar 2020]. Acesso em: http://www2.datasus.gov.br/DATASUS/index.php ?area $=0205 \&$ id $=6937$

4- Chair DLA, Berger S, Duff JP, Gonzales JC, Hunt EA, Joyner BL, et al. Part 11: Pediatric basic life support and cardiopulmonary resuscitation quality: 2015 American Heart Association guidelines update for cardiopulmonary resuscitation and emergency cardiovascular care. Circulation 2015;132(Suppl 2):S519-25. DOI: 10.1161/CIR.0000000000000265

5- Meira Júnior LE, Souza FM, Almeida LC, Veloso GGV, Caldeira AP. Avaliação de treinamento em suporte básico de vida para médicos e enfermeiros da atenção primária. Rev Bras Med Fam Comunidade 2016;11(38):1-10. DOI: 10.5712/rbmfc11(38)1231

6- Rosengarten L, Ban S. Basic life support for the child and infant. Br J Nurs. 2019;28(17):1118-22. DOI: $\quad 10.12968 /$ bjon.2019.28.17.1118. $\quad$ PMID: 31556731

7- Hasselager $A B$, Lauritsen $T$, Kristensen $T$, Bohnstedt C, Sonderskov C, Østergaard D, et al. What should be included in the assessment of 
laypersons' paediatric basic life support skills? Results from a Delphi consensus study. Scand J Trauma Resusc Emerg Med. 2018;26(1):9. DOI: 10.1186/s13049-018-0474-5

8- Hasselager $A$, Ostergaard $D$, Kristensen $T$, Sonderskov C, Bohnstedt C, Lauritsen TLB, et al. Assessment of laypersons' paediatric basic life support and foreign body airway obstruction management skills: A validity study. Scand J Trauma Resusc Emerg Med. 2018;26(1):73. DOI: 10.1186/s13049-018-0544-8

9- Ferreira RPN, Guedes HM, Oliveira SWD, Miranda JL. Simulação realística como método de ensino no aprendizado de estudantes da área da saúde. Rev Enferm Cent-Oeste Min. 2018;8:e2508. DOI: $10.19175 /$ recom.v8i0.2508

10- Bernoche C, Timerman S, Polastri TF, Giannetti NS, Siqueira AWS, Piscopo A, et al. Atualização da diretriz de ressuscitação cardiopulmonar e cuidados de emergência da sociedade brasileira de cardiologia - 2019. Arq Bras Cardiol. 2019;113(3):449-663. DOI: 10.5935/abc.20190203

11- Bajpai S, Chaturvedi HK, Bajpai R. Evaluation of inter-rater agreement and inter-rater reliability for observational data: An overview of concepts and methods. J Indian Acad Appl Psychol. 2015 [citado em 15 mar 2020]; 41(3):20-7. Acesso em: https://www.researchgate.net/publication/2734 51591 Evaluation of Inter-

Rater Agreement and InterRater Reliability for Observational Data An Overview of Concepts and Methods

12- Lima SG, Macedo LA, Vidal ML, Sá MPBO. Educação permanente em SBV e SAVC: Impacto no conhecimento dos profissionais de enfermagem. Arq Bras Cardiol. 2009;93(6):630-6. DOI: 10.1590/S0066-782X2009001200012

13- Tobase L, Peres HHC, Tomazini EAS, Teodoro SV, Ramos MB, Polastri TF. Basic life support: Evaluation of learning using simulation and immediate feedback devices. Rev Latino-Am Enferm. 2017;25:1-8. DOI: 10.1590/1518$\underline{8345.1957 .2942}$

14- Silva LGS, Costa JB, Furtado LGS, Tavares JB, Costa JLD. First aid and prevention of accidents in the school environment: Intervention in the educational unit. Enferm Foco 2017;8(3):25-9. DOI: 10.21675/2357- 707X.2017.v8.n3.893

15- Muffato PA, Baranhuk YT, Muffato CMMP, Oliveira BF, Mariano RS, Thiago Bertochi T, et al. Capacitação em suporte básico de vida cardiovascular em ambientes extra-hospitalares em cuiabá-MT. COORTE 2017 [citado em 15 mar
2020]; 7:9-17. Acesso em: http://revistacoorte.com.br/index.php/coorte/ar ticle/view/78

16- Cave DM, Aufderheide TP, Beeson J, Ellison A, Gregory A, Hazinski MF, et al. Importance and implementation of training in cardiopulmonary resuscitation and automated external defibrillation in schools: A science advisory from the American Heart Association. Circulation 2011;123(6):691-706.

DOI: 10.1161/CIR.0b013e31820b5328

17- Fernandes JMG, Leite ALS, Auto BSD, Lima JEG, Rivera IR, Mendonça MA. Ensino de suporte básico de vida para alunos de escolas pública e privada do ensino médio. Arq Bras Cardiol. 2014;102(6):593-601.

DOI: $10.5935 / a b c .20140071$

18- Diaz FBBS, Novais MEF, Alves KR, Cortes LP, Moreira TR. Conhecimento dos enfermeiros sobre - novo protocolo de ressuscitação cardiopulmonar. Rev Enferm Cent-Oeste Min. 2017;7:e1822. DOI: 10.19175/recom.v7i0.1822

19- Lyra PF, Cordeiro DEF, Gois ACR, Muniz FN, Leônidas GM, Rodrigues Sobrinho CRM. Programa de educação em reanimação cardiorrespiratória: Ensinando a salvar vidas. Rev Bras Educ Méd. 2012;36(4):570-3. DOI: $10.1590 / 50100-$ $\underline{55022012000600018}$

20- Azevedo CA, Silva GWS, Vale LD, Santos QG, Cassiano AN, Morais IF, et al. Educação continuada em enfermagem no âmbito da educação permanente em saúde: revisão integrativa de pesquisa. Rev Saúde Pesq. 2015;8(1):31-40. DOI: 10.17765/2176-9206.2015v8n1p131-140

21- Brkic $F$, Umihanic $S$, Altumbabic $H$, Ramas $A$, Salkic $A$, Umihanic $S$, et al. Death as a consequence of foreign body aspiration in children. Med Arch. 2018;72(3):220-3.

DOI: 10.5455/medarh.2018.72.220-223

Nota: este trabalho faz parte de monografia de conclusão do curso de graduação em Enfermagem.

Recebido em: 15/07/2020

Aprovado em: 07/12/2020

Endereço de correspondência:

Priscila Costa

Escola Paulista de Enfermagem. Rua Napoleão de Barros, 754. Vila Clementino, São Paulo. CEP: 04024-002

E-mail: priscila.costa@unifesp.br 